\section{THE ROLE OF LITHIUM IONS ON THE PASSIVATION OF AI-Li ALLOYS.}

\section{A. Travassos, C. Oliveira, C. M. Rangel Electroquimica de Materiais - DM \\ Instituto Nacional de Engenharia e Tecnologia Industrial}

$-$

\section{Abstract}

Al-Li alloy 8090 and a binary alloy with the same lithium contents (2.5 wt-\%) were studied in regard to their corrosion susceptibility in chloride containing solutions using electrochemical methods. Data were compared with results obtained for pure aluminium in the same experimental conditions

The effect of lithium ions on aluminium passivation has been demonstrated previously as particularly effective in alkaline solutions, with the obtained film having a protective character and an inhibitive action on the pitting nucleation process. In this work voltammetric and impedance spectroscopy studies were carried out in presence and absence of lithium ions, in alkaline solutions. Chloride ions were added in concentrations varying from $0.001 \mathrm{M}$ to $0.6 \mathrm{M}$ allowing pitting potentials to be determined and confirming the role the lithium ions in the promotion of corrosion resistance of aluminium and aluminium alloys.

Keywords: aluminium alloys, lithium, passivation, pitting corrosion

\section{Introdução}

A substituição de componentes estruturais em ligas ferrosas por ligas de aluminio na indústria de transportes, tem como base o decréscimo considerável no peso da estrutura que proporciona uma redução de consumo de combustível e dos custos globais de fabrico[1]. Sendo o lítio o metal mais leve e revelando boa solubilidade quando adicionado ao alumínio, a sua utilização como elemento de liga conduz a uma redução na densidade da liga e a um aumento no módulo de elasticidade e resistência mecânica. Assim, as ligas de Al-Li apresentam-se atractivas para uso na indústria de transportes.

É de salientar que a precipitação da fase metastável $\delta^{\prime}\left(\mathrm{Al}_{3} \mathrm{Li}\right)$, responsável pelo aumento da resistência mecânica das ligas de Al-Li e a elevada reactividade do lítio podem favorecer a corrosão da liga quando em contacto com soluções agressivas. A nucleação e crescimento dos precipitados $\delta^{\prime}\left(\mathrm{Al}_{3} \mathrm{Li}\right)$ dá-se em torno dos limites de grão da fase estável $\delta$ (AlLi) funcionando como zonas susceptíveis à corrosão localizada $[2,3]$.
Em trabalho anterior [4] foi demonstrado, que a presença de iões lítio em solução, em condições experimentais selectivas, pode conduzir à formação de um filme passivante. Neste trabalho, o efeito do lítio na susceptibilidade à corrosão localizada das ligas Al-Li foi avaliado em duas situações diferentes: uma associada à sua presença na liga, como a fonte potencial de iões lítio contribuindo para a formação do filme passivante [4,5], e outra envolvendo adição de lítio à solução aquosa em contacto com a liga. Utilizou-se para além da liga Al-Li com denominação 8090 ( teor em lítio de $\sim 2.5 \%$ em peso), uma liga binária com a mesma concentração em lítio e ainda, alumínio puro.

\section{Experimental}

O procedimento experimental está descrito com detalhe na referência [6] relativamente à preparação das amostras e à Voltametria Cíclica juntamente com a análise morfológica da superfície por Microscopia Electrónica de Varrimento(MEV) e ainda análise por Espectroscopia Auger (AES) e Espectroscopia de Fotoelectrões X (XPS). O carbonato de lítio em soluções de pH 11.2 e concentração de $0.05 \mathrm{M}$ foi utilizada como solução base. A composição da liga 8090 apresenta-se na tabela 1.

Tabela 1.- Composição da liga de Al- Li 8090

\begin{tabular}{|c|c|c|c|c|c|c|}
\hline Elemento & $\mathrm{Li}$ & $\mathrm{Cu}$ & $\mathrm{Mg}$ & $\mathrm{Zr}$ & $\mathrm{Fe}$ & $\mathrm{Si}$ \\
\hline$\%$ peso & 2.5 & 1.3 & 0.37 & 0.12 & 0.10 & 0.05 \\
\hline
\end{tabular}

\section{Resultados e Discussão}

O comportamento das ligas de Al-Li (8090), Al-Li (binária) e Al puro (99.999\%) foi avaliado em circuito aberto em soluções carbonatadas de pH 11.2, na presença e ausência de iões lítio em solução, figura 1. Em soluções $0.05 \mathrm{M} \mathrm{Li}_{2} \mathrm{CO}_{3}$, figura 1 (a), verificou-se, para todos os materiais, um deslocamento do potencial no sentido anódico, de cerca de $1 \mathrm{~V}$ sendo o potencial final de $\sim-0.550 \mathrm{~V}(\mathrm{ESC})$

A observação por MEV das superficies expostas permitiu verificar a existência de depósitos tipo filamento para as ligas e o alumínio puro após 100 horas de imersão, representando a figura 1 (b) a morfologia típica do depósito. 
Em soluções onde o ião lítio está ausente, figura 1 (c), os potenciais mantiveram-se bastante catódicos à excepção do caso da liga 8090, onde um deslocamento acentuado do potencial
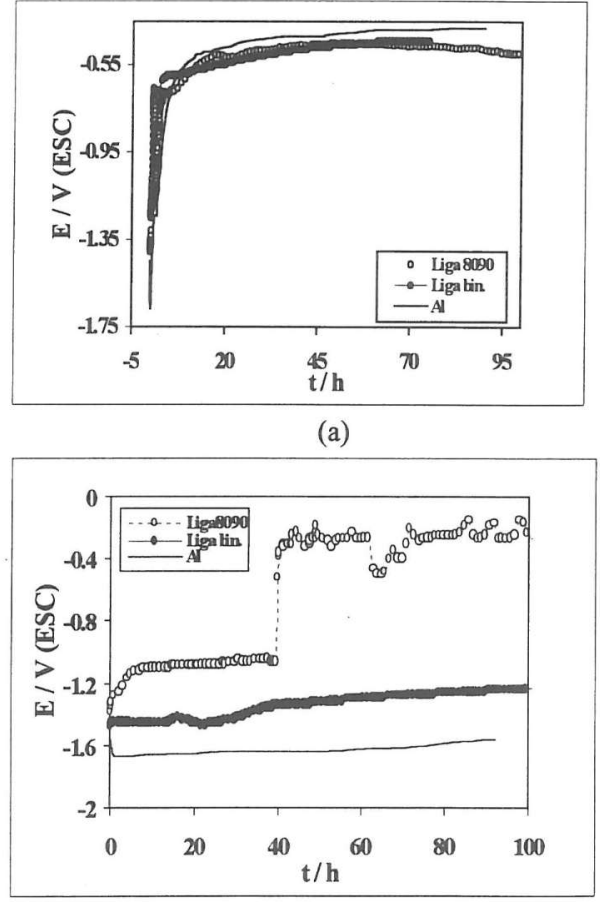

(b)

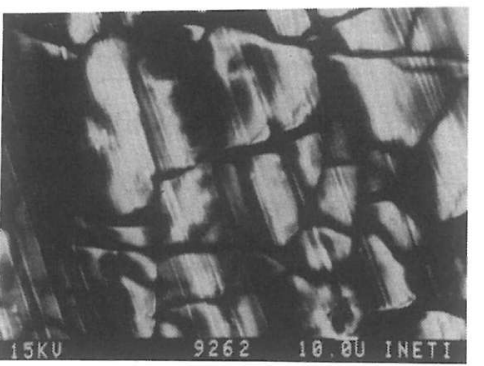

(d)

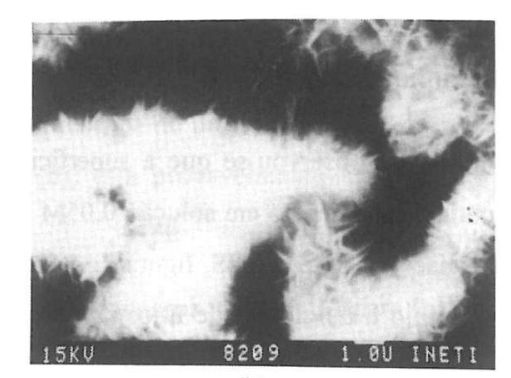

Figura 1-Perfil do potencial em circuito aberto como função do tempo de exposição para as ligas de Al-Li e Al em soluções carbonatadas, pH 11.2;

$0.05 \mathrm{M} \mathrm{Li}_{2} \mathrm{CO}_{3}$ (a); morfologia da superfície da liga binária/0.05 $\mathrm{M} \mathrm{Li}_{2} \mathrm{CO}_{3}(\mathrm{~b})$;

$0.05 \mathrm{M} \mathrm{Na}_{2} \mathrm{CO}_{3}$ (c); morfologia da superficie da liga $8090 / 0.05 \mathrm{M} \mathrm{Na}_{2} \mathrm{CO}_{3}$ (d)

no sentido anódico, após 40 horas de imersão, foi associado à presença de depósitos poligonais aderentes e espessos, figura 1(d). Neste último caso não foi observada a presença de corrosão no metal base, após remoção dos depósitos.

A influência dos iões cloreto na resistência à corrosão do Al e ligas Al-Li foi avaliada utilizando Voltametria Cíclica em soluções contendo também iões lítio. Para uma concentração em ião cloreto de $0.001 \mathrm{M}$ verificou-se a existência de densidades de corrente de passivação de " 10 $\mu \mathrm{A} . \mathrm{cm}^{-2}$, e a ausência de zonas de ruptura no voltamograma. O alumínio apresenta-se mais estável do que as ligas $\mathrm{Al}-\mathrm{Li}$ ao longo de todo o domínio de varrimento do potencial ( $-2 \mathrm{~V}$ a $+2 \mathrm{~V}$ (ESC)).
Foi observado um pico característico da formação de alumina a $-1.5 \mathrm{~V}(\mathrm{ESC})$ comúm as ligas, embora desfasado para potencias mais anódicos. Para a liga 8090, o pico transpassivo que aparece nas curvas de polarização obtidas em $0.05 \mathrm{M} \mathrm{Li}_{2} \mathrm{CO}_{3}$ ou $0.05 \mathrm{M} \mathrm{Na}_{2} \mathrm{CO}_{3}$, na ausência de iões cloretos, foi também observado. Quanto à liga binária verifica-se estabilidade no sentido anódico, mas após inversão do potencial foram evidentes flutuações na densidade de corrente indicativas de corrosão localizada [7].

Por MEV observou-se que a superficie das amostras tinha o mesmo aspecto filamentoso que o obtido por imersão em solução $0.05 \mathrm{M} \mathrm{Li}_{2} \mathrm{CO}_{3}$. Para avaliar da composição do filme recorreu-se a análise por AES e XPS, figura 2. A liga 8090 e a liga binária revelaram a existência de óxido de alumínio e de cloreto de lítio. Para o Al só foram detectados picos correspondentes ao alumínio, oxigénio e cloro, correspondendo possivelmente a uma mistura de $\mathrm{Al}_{2} \mathrm{O}_{3}$ e $\mathrm{AlCl}_{3}$. O facto de neste último caso o lítio não ter sido detectado sugere, por um lado, a possibilidade de se encontrar presente em quantidades muito pequenas e, por outro, que as ligas Al-Li podem funcionar como fonte de iões lítio passíveis de ser incorporados no filme [5]
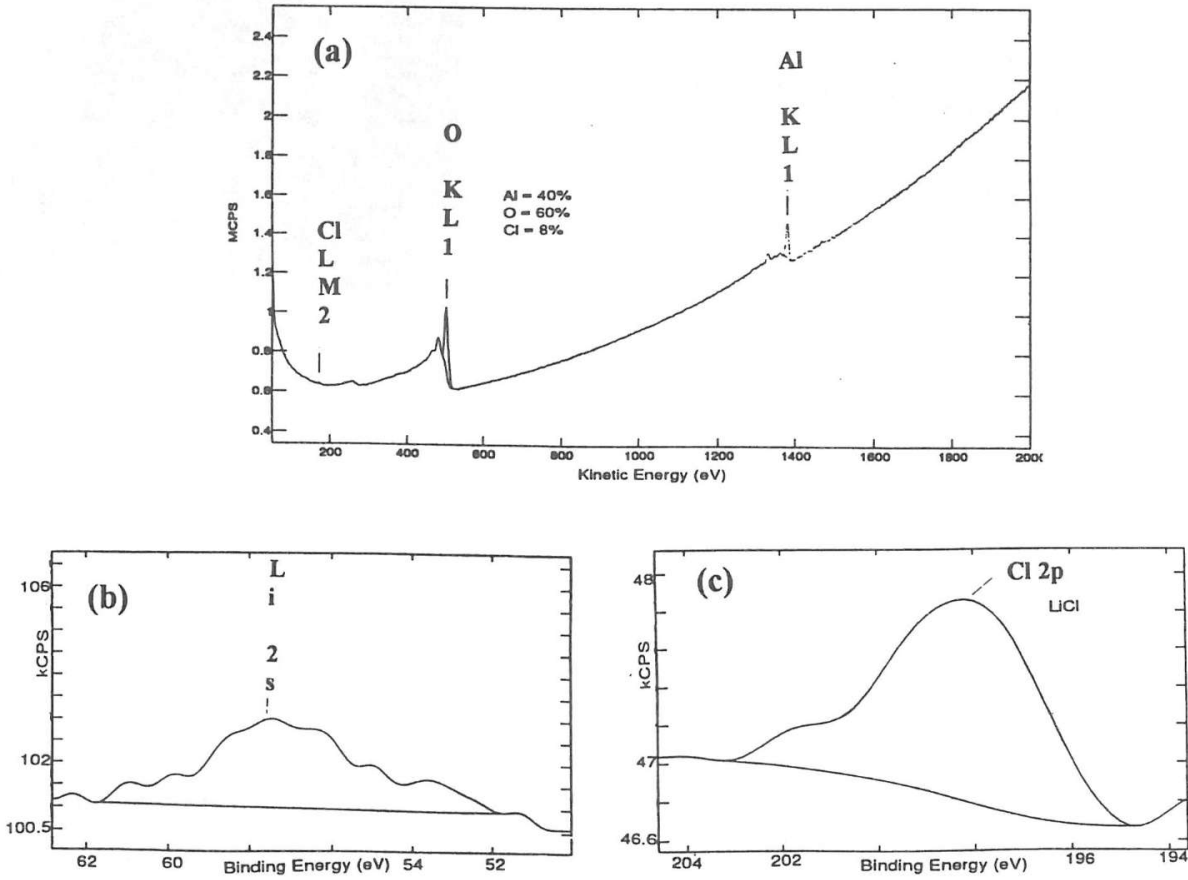

Figura 2 - Espectro AES para a liga 8090 após curvas de polarização cíclica em solução mista de $0.05 \mathrm{M} \mathrm{Li}_{2} \mathrm{CO}_{3}+0.001 \mathrm{M} \mathrm{NaCl}, \mathrm{pH} 11.2, \mathrm{v}=1 \mathrm{mV} \cdot \mathrm{s}^{-1}$ (a) Espectro XPS para Li 1s (b); espectro XPS para $\mathrm{Cl} 2 \mathrm{p}(\mathrm{c})$ 
Quando em soluções carbonatadas isentas de lítio $\left(0.05 \mathrm{M} \mathrm{Na}_{2} \mathrm{CO}_{3}, \mathrm{pH} 11.2\right)$ se adiciona $\mathrm{NaCl}$ $(0.001 \mathrm{M})$, obtém-se à superficie das amostras após Voltametria Cíclica, depósitos com forma de placas rectangulares relativamente espessas observáveis por MEV, que após remoção mostram a presença de corrosão localizada, mais notória na liga 8090. Depósitos semelhantes já tinham sido observados após imersão em solução $0.05 \mathrm{M} \mathrm{Na}_{2} \mathrm{CO}_{3}$, figura 1. As amostras ensaiadas revelaram ausência de lítio à superficie. Apenas foram detectados por XPS, figura 3 (a), os picos correspondentes ao $\mathrm{Al}(2 \mathrm{p}), \mathrm{O}(1 \mathrm{~s})$ e $\mathrm{Cl}(2 \mathrm{p})$, sendo possivel a presença de um óxido de $\mathrm{Al}$ e de um cloreto de Al. A presença de uma ligação $\mathrm{C}-\mathrm{O}$, figura 3 (b), está provavelmente associada à depósitos contendo carbonatos

Com o aumento na concentração de iões cloreto nas soluções de carbonatos, de $0.001 \mathrm{M}$ para $0.1 \mathrm{M}$, verificou-se, por Voltametria Cíclica, ruptura do filme apenas no caso das amostras ensaiadas em meio isento de lítio, como é evidente na tabela 2. Em geral, os valores dos potenciais de picada (Ep) são mais anódicos para a liga 8090 do que para o Al e a liga binária. Para uma concentração em $\mathrm{NaCl}$ de $0.6 \mathrm{M}$ em soluções de $0.05 \mathrm{M} \mathrm{Li}_{2} \mathrm{CO}_{3}$ e $0.05 \mathrm{M} \mathrm{Na}_{2} \mathrm{CO}_{3}$ a corrosão localizada foi evidente em ambos os casos. Observou-se, também, que há um efeito "inibidor" da corrosão atribuivel ao ião carbonato. Este efeito será objecto de um mais detalhado estudo.

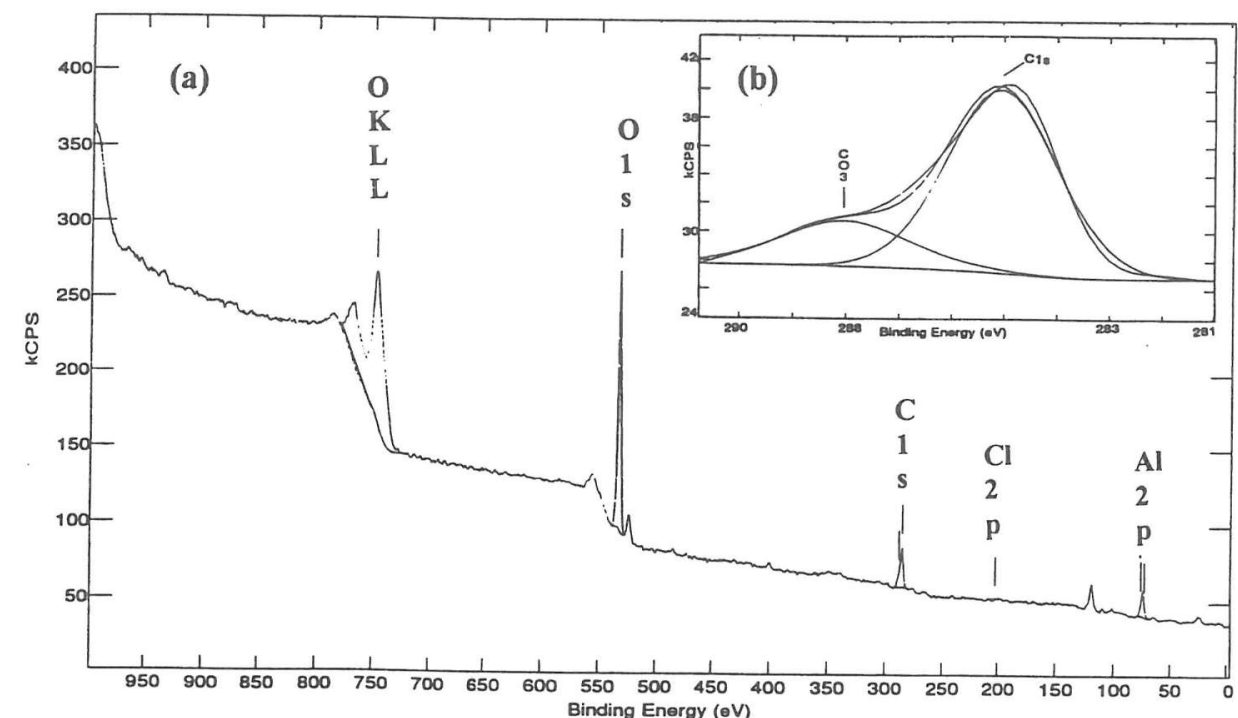

Figura 3 - Espectro XPS para a liga binária após curvas de polarização cíclica em solução mista de $0.05 \mathrm{M} \mathrm{Na}_{2} \mathrm{CO}_{3}+0.001 \mathrm{M} \mathrm{NaCl}, \mathrm{pH} 11.2, \mathrm{v}=1 \mathrm{mV} \mathrm{s}^{-1}$ (a), espectro XPS para $\mathrm{C} 1 \mathrm{~s}(\mathrm{~b})$.
Tabela 2 - Potenciais de picada (Ep) para Al puro e ligas Al-Li obtidos por voltametria, em solucões carbonatadas na presença e ausência de lítio, $\mathrm{V}=1 \mathrm{mVs} \mathrm{s}^{-1}$, $\mathrm{pH} 11.2$.

\begin{tabular}{|c|c|c|c|c|c|}
\hline \multirow{2}{*}{ Amostra } & \multicolumn{4}{|c|}{ Solução } & \multirow{2}{*}{$\begin{array}{c}\mathbf{E}_{\mathrm{p}} \\
(\mathrm{V} / \mathrm{ESC})\end{array}$} \\
\hline & $\begin{array}{r}0.05 \mathrm{M} \\
\mathrm{Li}_{2} \mathrm{CO}_{3} \\
\end{array}$ & $\begin{array}{c}0.05 \mathrm{M} \\
\mathrm{Na}_{2} \mathrm{CO}_{3} \\
\end{array}$ & $\begin{array}{l}0.1 \mathrm{M} \\
\mathrm{NaCl} \\
\end{array}$ & $\begin{array}{l}0.6 \mathrm{M} \\
\mathrm{NaCl} \\
\end{array}$ & \\
\hline \multirow{4}{*}{$\mathrm{Al}$ puro } & $\mathrm{x}$ & \multirow{3}{*}{$\mathrm{X}$} & \multirow{2}{*}{$\begin{array}{l}X \\
X\end{array}$} & \multirow{4}{*}{$\begin{array}{l}X \\
X\end{array}$} & - \\
\hline & \multirow[b]{2}{*}{$\mathrm{x}$} & & & & -0.245 \\
\hline & & & \multirow[b]{3}{*}{$\mathrm{X}$} & & -0.540 \\
\hline & & \multirow[t]{2}{*}{$\mathrm{x}$} & & & -0.515 \\
\hline \multirow{4}{*}{$\begin{array}{c}\text { Al-Li (Liga } \\
\text { binária) }\end{array}$} & $\mathrm{X}$ & & & & - \\
\hline & & \multirow{2}{*}{$\mathrm{x}$} & \multirow[t]{2}{*}{$\mathrm{X}$} & & +0.260 \\
\hline & \multirow[t]{2}{*}{$\mathrm{X}$} & & & $\mathrm{X}$ & -0.620 \\
\hline & & \multirow[t]{2}{*}{$\mathrm{x}$} & \multirow{3}{*}{$\begin{array}{l}X \\
X\end{array}$} & $\mathrm{X}$ & -0.530 \\
\hline \multirow{4}{*}{ Al-Li 8090} & $\mathrm{x}$ & & & & - \\
\hline & & \multirow[t]{2}{*}{$\mathrm{x}$} & & \multirow[b]{2}{*}{$\mathrm{X}$} & +0.250 \\
\hline & \multirow[t]{2}{*}{$\mathrm{x}$} & & & & -0.300 \\
\hline & & $\mathrm{x}$ & & $\mathrm{x}$ & -0.400 \\
\hline
\end{tabular}

\section{Conclusões}

- A formação de um filme passivante foi evidenciado na presença de iões lítio para o aluminio e ligas Al-Li, pelo enobrecimento acentuado dos potenciais em circuito aberto e pela não existência de zonas de ruptura nos voltamogramas.

Os iões lítio em solução, na forma de carbonato de lítio, inibiram a nucleação de picadas na presença de iões cloreto para concentrações até $0.1 \mathrm{M}$ para polarizações com limite superior de $+2 \mathrm{~V}(\mathrm{ESC})$ 


\section{Referências}

[1] - A. Conde, J.de Damborenea, J. Mater. Sci., 31(1996) 4921

[2] - R. Ambat, E.S. Dwarakadasa, Corros. Sci., 33(1992),681

[3] - M. Lieblich, M. Torralba, Rev. Metal. Madrid, 26(1990)314

[4] - C. M. Rangel, M .A. Travassos, Corros. Sci., 33(1992) 327

[5] - K. S. N. Murthy, E.S. Dwarakadasa, Br. Corros. J., 30(1995)111

[6] - M. A. Travassos, C. Oliveira, C. M. Rangel, Portugaliae Electroch. Acta, a submeter (1999)

[7] - C. Oliveira, "Passivação de Ligas de Al-Li e a sua Estabilidade em Meios Agressivos", Tese para a obtenção do grau de Licenciatura em Química Tecnológica da FCL, (1998) 